\title{
A LIFTING THEOREM AND ANALYTIC OPERATOR ALGEBRAS
}

\author{
TAKAHIKO NAKAZI
}

(Communicated by Paul S. Muhly)

Dedicated to Professor Shozo Koshi on his sixtieth birthday

\begin{abstract}
Let $K$ be a complex Hilbert space and $H$ a closed subspace. It is shown that if a $2 \times 2$ selfadjoint operator matrix $\mathscr{T}$ with positive diagonals on $K \oplus K$ is positive on $H \oplus H^{\perp}$, then there exists a $2 \times 2$ operator matrix $\tilde{\mathscr{T}}$ with the same diagonals such that $\tilde{\mathscr{T}}$ is positive on $K \oplus K$ and $\mathscr{T}$ is the restriction of $\tilde{\mathscr{T}}$ to $H \oplus H^{\perp}$. When $\mathscr{T}$ is in a von Neumann algebra, we consider the problems of finding $\mathscr{T}$ in the same algebra. This lifting theorem has applications to weighted norm inequalities for conjugation operators on analytic operator algebras.
\end{abstract}

1. Introduction. Let $\mathscr{L}(K)$ be the set of all bounded linear operators on a complex Hilbert space $K$ and $\mathscr{B}$ a von Neumann algebra on $K$. Let $H$ be the closed subspace of $K$ and $\mathscr{A}$ a (perhaps nonselfadjoint) weakly closed subalgebra of $\mathscr{B}$ which has $H$ as an invariant subspace. We study $2 \times 2$ operator matrices $\mathscr{T}=\left(T_{i j}\right)$ on $K \oplus K$ where $T_{i j} \in \mathscr{B}(i, j=1,2), T_{11} \geq 0, T_{22} \geq 0$ and $T_{21}^{*}=T_{12}$. $[\mathscr{B}]$ denotes the set of such operator matrices $\mathscr{T}$. [ $\mathscr{A}]_{0}$ denotes the subset of $[\mathscr{B}]$ such that $T_{12} \in \mathscr{A}$ and $T_{11}=T_{22}=0$.

Let us denote

$$
\mathscr{T}\left[f_{1}, f_{2}\right]=\sum_{i, j=1}^{2}\left(T_{i j} f_{i}, f_{j}\right) .
$$

If $\mathscr{T}$ satisfies $\mathscr{T}\left[f_{1}, f_{2}\right] \geq 0$ for all $f_{1}$ in $H$ (resp. $K$ ) and $f_{2}$ in $H^{\perp}$ (resp. $K$ ), then $\mathscr{T}$ is said to be positive on $H \oplus H^{\perp}$ (resp. $K \oplus K$ ) where $H^{\perp}$ is the orthogonal complement of $H$ in $K . \mathscr{T}$ is positive on $H \oplus H^{\perp}$ if and only if compression $\mathscr{P} \mathscr{T}$ of $\mathscr{T}$ to $H \oplus H^{\perp}$ is positive where $\mathscr{P}$ is the orthogonal projection of $K \oplus K$ onto $H \oplus H^{\perp}$.

When $\mathscr{T} \in[\mathscr{B}]$ and $\mathscr{T}$ is positive on $H \oplus H^{\perp}$, we wish to $\tilde{\mathscr{T}}$ in $\mathscr{T}+[\mathscr{A}]_{0}$ which is positive on $K \oplus K$. Of course then $\mathscr{T}\left[f_{1}, f_{2}\right]=\tilde{\mathscr{T}}\left[f_{1}, f_{2}\right]$ for all $f_{1}$ in $H$ and $f_{2}$ in $H^{\perp}$ or equivalently $\mathscr{P} \mathscr{T}\left|H \oplus H^{\perp}=\mathscr{P} \mathscr{T}\right| H \oplus H^{\perp}$. This problem is related with a lifting theorem of Cotlar and Sadosky [4] for the disk algebra and a generalized lifting theorem of the author and Yamamoto [10] for a general uniform algebra. In the special case, they consider the problem above when $\mathscr{B}$ is commutative. In our main theorm in $\S 2$, we do not assume that $\mathscr{B}$ is commutative and we establish a

Received by the editors December 9, 1987.

1980 Mathematics Subject Classification (1985 Revision). Primary 47A20, 47A15, 47D25.

Key words and phrases. Commutant lifting, Arveson distance formula, factorization, HelsonSzegö theorem.

This research was partially supported by Grant-in-Aid for Scientific Research, Ministry of Education. 
lifting theorem. We then exhibit some examples in $\S 3$ and obtains two operator theoretic type Helson-Szegö in $\S 4$ (cf. [6, 7]).

2. General lifting theorem. Let $\mathscr{F}$ be the subset of lat $\mathscr{A}$, the lattice of all $\mathscr{A}$-invariant projections, then for any $T$ in $\mathscr{B}$

$$
\operatorname{dist}(T, \mathscr{A}) \geq \sup _{P \in \mathscr{F}}\|(1-P) T P\| .
$$

Many concrete examples satisfy the following conditions (I) and (II) (see $\S 3$ ).

(I) There exists a subset $\mathscr{F}$ in lat $\mathscr{A}$ such that for any $T$ in $\mathscr{B}$

$$
\operatorname{dist}(T, \mathscr{A})=\sup _{P \in \mathscr{F}}\|(1-P) T P\| .
$$

(II) Every invertible positive operator in $\mathscr{B}$ has the form $A^{*} A=B B^{*}$, for some invertible $A$ and $B$ in $\mathscr{A}$.

(I) is a kind of Nehari's theorem for Hankel operators (cf. [12, 8, 3, 13 and 9]). (II) is a factorization of Szegö-Cholesky type (cf. [5, 3 and 13]). The following is a lifting theorem under the conditions (I) and (II). Then we say that $(\mathscr{B}, \mathscr{A}, \mathscr{F})$ has the lifting property when the conclusion of the theorem holds.

THEOREM. Let $(\mathscr{B}, \mathscr{A}, \mathscr{F})$ satisfy the condition (I) and (II). If $\mathscr{T}$ in $[\mathscr{B}]$ is positive on $P K \oplus(1-P) K$ for every $P$ in $\mathscr{F}$, then there exists $\tilde{\mathscr{T}}$ in $\mathscr{T}+[\mathscr{A}]_{0}$ which is positive on $K \oplus K$.

ProOF. $\mathscr{T}=\left[T_{i j}\right]$ is positive on $P K \oplus(1-P) K$ if and only if for any $f \in P K$ and $g \in(1-P) K$

$$
\left|\left(T_{12} f, g\right)\right|^{2} \leq\left(T_{11} f, f\right)\left(T_{22} g, g\right) .
$$

By the hypothesis (II), for any positive integer $n$ there exist two invertible operators $A_{n}$ and $B_{n}$ in $\mathscr{A}$ such that

$$
T_{11}+1 / n=A_{n}^{*} A_{n} \quad \text { and } \quad T_{22}+1 / n=B_{n} B_{n}^{*} .
$$

Since $P \in$ lat $\mathscr{A}, A_{n} P K=P K$ and $B_{n}^{*}(1-P) K=(1-P) K$, and hence for any $f \in P K$ and $g \in(1-P) K$

$$
\left|\left(B_{n}^{-1} T_{12} A_{n}^{-1} f, g\right)\right|^{2} \leq(f, f)(g, g) .
$$

By the hypothesis (I), for any positive number $\varepsilon$ there exists $D_{n, \varepsilon}$ in $\mathscr{A}$ such that

$$
\left\|B_{n}^{-1} T_{12} A_{n}^{-1}+D_{n, \varepsilon}\right\| \leq \sup \left\|(1-P) B_{n}^{-1} T_{12} A_{n}^{-1} P\right\|+\varepsilon \leq 1+\varepsilon .
$$

Since $\mathscr{A}$ is weakly closed, there exists $D_{n}$ in $\mathscr{A}$ such that

$$
\left\|B_{n}^{-1} T_{12} A_{n}^{-1}+D_{n}\right\| \leq 1 \text {. }
$$

Put $S_{n}=B_{n} D_{n} A_{n}$, then $S_{n}$ is in $\mathscr{A}$ and for any $F, G \in K$

$$
\left|\left(\left(T_{12}+S_{n}\right) F, G\right)\right|^{2} \leq\left(\left(T_{11}+1 / n\right) F, G\right)\left(\left(T_{22}+1 / n\right) F, G\right) .
$$

Again using the weakly closedness of $\mathscr{A}$, there exists $S$ in $\mathscr{A}$ such that for any $F$, $G \in K$

$$
\left|\left(\left(T_{12}+S\right) F, G\right)\right|^{2} \leq\left(T_{11} F, G\right)\left(T_{22} F, G\right)
$$

and the theorem follows. 
3. $(\mathscr{B}, \mathscr{A}, \mathscr{F})$ with the lifting property. The theorem shows that if $(\mathscr{B}, \mathscr{A}, \mathscr{F})$ satisfies (I) and (II) then it has a lifting property. Hence it is sufficient to check (I) and (II) in the following examples.

(1) Let $\mathscr{B}=\mathscr{L}(K), P$ an orthogonal projection and

$$
\mathscr{A}=\{A \in \mathscr{L}(K): P A P=A P\} .
$$

It is clear that, for any $T \in \mathscr{L}(K), \operatorname{dist}(T, \mathscr{A})=\|(1-P) T P\|$. It is well known that if $T$ is invertible then $T=A^{*} A=B B^{*}$ for some invertible $A$ and $B$ in $\mathscr{A}$ (see $[1$, Chapter I, 13]). The theorem shows that $(\mathscr{L}(K), \mathscr{A}, P)$ has a lifting property.

(2) In (1) let $U$ be a bilateral shift operator on $K$ with $U P=P U P$. Suppose $\bigcap_{n=0}^{\infty} U^{n}(P K)=\{0\}$ and $\bigcup_{n=0}^{\infty} U^{* n}(P K)$ is dense in $K$. If $\mathscr{T} \in[\mathscr{L}(K)], \mathscr{T}=$ $\left[T_{i j}\right]$ and $T_{i j}$ commutes with $U$ for $i, j=1,2$, then we can get a positive lifting operator $\tilde{\mathscr{T}}=\left[\tilde{T}_{i j}\right]$ in $\mathscr{T}+[\mathscr{A}]_{0}$ such that $\tilde{T}_{i j}$ commutes with $U$. This is a kind of commuting lifting (see [14]). In fact let $\mathscr{B}_{1}=\{T \in \mathscr{L}(K): U T=T U\}$, $\mathscr{A}_{1}=\{A \in \mathscr{A}: U A=A U\}$ and $\mathscr{F}=\{P\}$, then (I) and (II) are true, too $[12$ and 5]. The theorem shows that $\left(\mathscr{B}_{1}, \mathscr{A}_{1}, P\right)$ has the lifting property.

(3) Let $\mathscr{B}$ be a factor with faithful semifinite normal trace $\tau$ and let $\mathscr{E}$ be a complete nest of selfadjoint projections in $\mathscr{B}$. We write Alg $\mathscr{E}$ for the nest algebra associated with $\mathscr{E}$, and let

$$
\mathscr{A}=\mathscr{B} \cap \operatorname{Alg} \mathscr{E} .
$$

(I) with $\mathscr{F}=\mathscr{E}$ is a variant of Arveson's distance formula, that is,

$$
\operatorname{dist}(T, \mathscr{A})=\sup _{P \in \mathscr{F}}\|(1-P) T P\|
$$

(see [3]). Assuming $\mathscr{E}$ is well-ordered, (II) is valid by [13]. The theorem shows that $(\mathscr{B}, \mathscr{A}, \mathscr{F})$ has the lifting property.

(4) In (3) let

$$
L^{p}=L^{p}(\mathscr{B}, \tau), \quad 1 \leq p \leq \infty,
$$

be the usual noncommutative Lebesgue spaces and define the noncommutative Hardy space

$$
H^{p}=H^{p}(\mathscr{B}, \mathscr{E}, \tau)
$$

to be the closed subspace of $L^{p}$ of elements $T$ for which $(1-P) T P=0$ for all $P \in \mathscr{E}$. In particular $L^{\infty}=\mathscr{B}$ and $H^{\infty}=\mathscr{A}$. Let $P_{0}$ be the orthogonal projection from $L^{2}$ onto $H^{2}$ and $\mathscr{F}=\left\{P_{0}\right\}$. By [13], with $\mathscr{F}=\left\{P_{0}\right\}$ property (I) holds, that is,

$$
\operatorname{dist}\left(T, H^{\infty}\right)=\left\|\left(1-P_{0}\right) T P_{0}\right\| \text {. }
$$

(Here $T$ acts by left multiplication.)

Assuming $\mathscr{E}$ is well-ordered, (II) is valid by [13] again. The theorem shows that $\left(L^{\infty}, H^{\infty}, P_{0}\right)$ has the lifting property.

(2) is known and was shown by Arocena and Cotlar [2]. However our proof is different from theirs and more operator theoretic. In (2) if $U$ is a bilateral shift with multiplicity 1 , then the lifting theorem is classical and was shown by Cotlar and Sadosky (cf. [4]). We can show this using (3) and [3, Proposition 5.1].

4. Applications. In this section, using the theorem we obtain the partial generalizations of the Helson-Szegö theorem and a theorem of Koosis as in [4 or 10]. 
COROLlaRY 1. Let $(\mathscr{B}, \mathscr{A}, \mathscr{F})$ satisfy the conditions (I) and (II). Let $W$ be a positive operator in $\mathscr{B}$. Then there exists a constant $C$ such that for every $P \in \mathscr{F}$.

$$
(W f, f) \leq C(W(f+g), f+g)
$$

for all $f \in P K$ and all $g \in(1-P) K$ if and only if there exists a nonzero operator $D$ in $\mathscr{A}$ such that

$$
\left\|W^{-1 / 2}(W+D) W^{-1 / 2}\right\| \leq \rho<1,
$$

where $W^{-1 / 2}$ is defined to be the (possibly unbounded) inverse of $W^{1 / 2}$ restricted to the orthocomplent of the kernel of $W^{1 / 2}$.

Proof. $(W f, f) \leq C(W(f+g), f+g)$ if and only if

$$
|(W f, g)|^{2} \leq\left(1-C^{-1}\right)(W f, f)(W g, g) .
$$

Hence the weighted norm inequality with $C$ holds if and only if $\mathscr{T}=\left[T_{i j}\right]$ in $[\mathscr{B}]$ is positive on $P K \oplus(1-P) K$ for every $P \in \mathscr{F}$ where $T_{11}=\rho W, T_{22}=T_{12}=T_{21}=W$ and $\rho=1-C^{-1}$. By the theorem, if the weighted norm inequality holds then there exists $\tilde{\mathscr{T}}$ in $\mathscr{T}+[\mathscr{A}]_{0}$ which is positive on $K \oplus K$. Hence there exists an operator $D$ in $\mathscr{A}$ such that

$$
|((W+D) F, G)|^{2} \leq \rho(W F, F)(W G, G)
$$

for any $F, G \in K$. This implies $\left\|W^{-1 / 2}(W+D) W^{-1 / 2}\right\| \leq \rho$. The converse is not difficult.

COROLlARY 2. Suppose that $(\mathscr{B}, \mathscr{A}, \mathscr{F})$ satisfies the conditions (I) and (II). Let $W$ be a positive operator in $\mathscr{B}$. Then there exists a nonzero positive operator $U \leq W$ such that for every $P \in \mathscr{F}$

$$
(U f, f) \leq(W(f+g), f+g)
$$

for all $f \in P K$ and all $g \in(1-P) K$ if and only if there exists a nonzero operator $D$ in $\mathscr{A}$ such that

$$
\left\|W^{-1 / 2}(W+D) W^{-1 / 2}\right\| \leq 1
$$

and $W \neq\left(W^{-1 / 2}(W+D)\right)^{*}\left(W^{-1 / 2}(W+D)\right)$.

PROOF. The part of 'only if' can be proved as in Corollary 1. If there exists a nonzero positive operator $U$ then $\mathscr{T}=\left[T_{i j}\right] \in[\mathscr{B}]$ is positive on $P K \oplus(1-P) K$ for every $P \in \mathscr{F}$ where $T_{11}=W-U, T_{22}=T_{12}, T_{21}=W$. By the theorem, there exists an operator $D$ in $\mathscr{A}$ such that

$$
|((W+D) F, G)|^{2} \leq((W-U) F, F)(W G, G)
$$

for any $F, G \in K$. This implies $\left\|W^{-1 / 2}(W+D) W^{-1 / 2}\right\| \leq 1$ and

$$
\left\|W^{-1 / 2}(W+D) F\right\|^{2}=\sup _{G} \frac{|((W+D) F, G)|^{2}}{(W G, G)} \leq((W-U) F, F) \leq(W F, F)
$$

(see $\left[1\right.$, Chapter I]). Thus $W-\left(W^{-1 / 2}(W+D)\right)^{*}\left(W^{-1 / 2}(W+D)\right) \leq U$. For the converse, $U=W-\left(W^{-1 / 2}(W+D)\right)^{*}\left(W^{-1 / 2}(W+D)\right)$ satisfies the weighted norm inequalities.

By (2) in $\S 3$, Corollary 1 is the Helson-Szegö theorem [6] when the weight function $W$ is bounded. Similarly Corollary 2 shows the Koosis theorem [7] when the weight function $W$ is bounded. However in the classical case if there exists a nonzero operator $D$ in $\mathscr{A}$ such that $\left\|W^{-1 / 2}(W+D) W^{-1 / 2}\right\| \leq 1$ then $W \neq\left(W^{-1 / 2}(W+D)\right)^{*}\left(W^{-1 / 2}(W+D)\right)$. 


\section{REFERENCES}

1. T. Ando, Topics on operator inequalities, Lectur€ Note, Sapporo, 1978.

2. R. Arocena and M. Cotlar, Dilation of generalized Toeplitz kernels and some vectorial moment and weighted problems, Harmonic Analysis (F. Ricci and G. Weiss, eds.), Lecture Notes in Math., vol. 908, Springer-Verlag, Berlin, Heidelberg and New York, 1982, pp. 169-186.

3. W. Arveson, Interpolation problems in nest algebras, J. Funct. Anal. 20 (1975), 208-233.

4. M. Cotlar and C. Sadosky, On the Helson-Szegö theorem and a related class of modified Toeplitz kernels, Proc. Sympos. Pure Math. vol. 35, Amer. Math. Soc., Providence, R. I., 1979, pp. 383-407.

5. R. Gellar and L. Page, Inner-auter factorizations of operators, J. Math. Anal. Appl. 61 (1977), 151-158.

6. H. Helson and G. Szegö, A problem in prediction theory, Ann. Mat. Pura Appl. 51 (1960), 107-138.

7. P. Koosis, Moyennes quadratiques pondérées de fonctions périodiques et de leurs conjuguées harmoniques, C. R. Acad. Sci. Paris 291 (1980), 255-257.

8. P. S. Muhly, Compact operators in the commutant of a contraction, J. Funct. Anal. 8 (1971), 197-224.

9. T. Nakazi, Norms of Hankel operators and uniform algebras, Trans. Amer. Math. Soc. 299 (1987), 573-580.

10. T. Nakazi and T. Yamamoto, $A$ lifting theorem and uniform algebras, Trans. Amer. Math. Soc. 305 (1988), 79-94.

11. Z. Nehari, On bounded bilinear forms, Ann. of Math. 65 (1957), 153-162.

12. L. B. Page, Bounded and compact vectorial Hankel operators, Trans. Amer. Math. Soc. 150 (1970), 529-539.

13. S. C. Power, Factorizations in analytic operator algebras, J. Funct. Anal. 67 (1986), 413-432.

14. B. Sz.-Nagy and C. Foias, Dilation des commutants d'opérateurs, C. R. Acad. Sci. Paris Sér. A-B 266 (1968), A493-A495.

Department of Mathematics, Faculty of Science, Hokkaido University, SAPPORO 060, JAPAN 